\title{
Computer-based preference models for generic multidimensional problem sets
}

\author{
JAMES C. MUNDT \\ University of Wisconsin, Madison, Wisconsin
}

\begin{abstract}
Alternative actions or objects can be characterized by differences among defining attributes or dimensions. Making choices among these alternatives is often a complex and difficult task, particularly when the number of alternatives or defining attributes is large or the time to decide is limited. This paper describes two programs that allow users to define a multidimensional problem domain and derive a multiplicative utility function that quantitatively describes their judgment policy within that domain. It also discusses potential applications for the derived preference models.
\end{abstract}

Making decisions among alternatives that vary widely across descriptive attributes is often a complex and cognitively difficult task. Applying a coherent, consistent decision-making policy may be virtually impossible under even moderate time constraints. In 1961, Yntema and Torgerson recognized both the potential for computers to aid decision-makers in difficult situations and the problems of representing human judgment with a programmable policy. Since then, the discipline of decision analysis, drawing from economics, statistics, psychology, and other fields, has developed methods for eliciting and mathematically representing expressed preferences among multiattribute alternatives (for general references see Keeney \& Raffia, 1976; von Winterfeldt \& Edwards, 1986).

This paper describes two recent programs that allow a user to define a problem domain and construct a quantitative model of the user's judgmental policy applied to that domain. The first program (DEFINE) prompts the user to identify and scale the attributes describing the problem domain and then generates exemplars, varying in value across these attributes, for the user to rate. The second program (SOLVE) derives a multiplicative utility function from ratings supplied by the user that represents his or her judgmental policy. Both programs were written in Turbo Pascal (version 3.0) and run on IBMcompatible computers using CGA graphics.

\section{PROGRAM DEFINE}

DEFINE prompts the user to define a problem set space, or to load a previously defined problem space, and then generates exemplars from this space. The user's rat-

This research was supported by Grant AA6093 from the National Institute on Alcohol Abuse and Alcoholism, awarded to L. E. Ross. The author would also like to recognize Dennis Fryback for providing the mathematical method needed to accomplish the method of gradient descent used to derive the preference models. Address correspondence to James C. Mundt, Department of Psychology, University of Wisconsin, Madison, WI 53706. ings and the attribute values of these exemplars are stored for model construction. In order to make the program description explicit, below is an example of a user interested in developing a model for evaluating automobiles.

\section{Defining the Problem Set Space}

It will be assumed here that the user wishes to define the problem set space for cars, but the program does allow previously defined problem dimensions and attribute levels to be read from a disk, and for response elicitation to commence immediately.

The first prompt for defining a new problem domain asks the user to specify the number of dimensions or attributes (up to 15) that will be used to describe each exemplar, and to label each dimension (up to 10 characters). It will be assumed that the hypothetical user wishes to evaluate automobiles with respect to 8 dimensions: price, gas mileage (MPG), comfort, color, safety, maintenance costs (upkeep), size, and make. After the dimensions have been specified, the user is asked to scale each dimension either quantitatively or as object- or phrase-anchored scales.

Quantitative scales. For dimensions that can be quantitatively scaled, such as price or MPG, the user is prompted to provide the numeric range (minimum and maximum) to be observed in the problem domain being defined. For example, if the user was interested only in new cars (used cars would probably require additional dimensions for evaluation, such as odometer reading, condition of tires, extent of body rust, etc.), then he or she might decide that $\$ 5,000$ was the lowest price for which a new automobile might be found, and that $\$ 15,000$ was the upper limit of his or her financial ability. Similarly, gas mileage might be expected to range between 15 and 55 MPG. After specifying the quantitative range, the user is asked if it is desirable for the attribute values to be large ("No" for price, "Yes" for MPG). The user is then asked to provide scale values between 0 and 100 to numeric values that are one-fourth, one-half, and three-fourths of the way between the minimum and maximum limits 


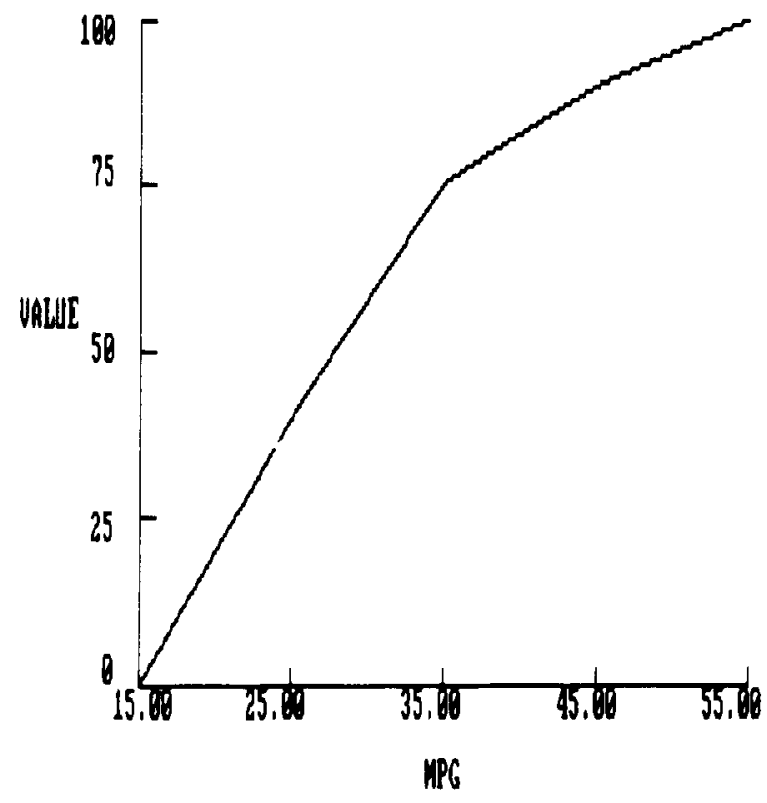

Figure 1. A hypothetical transfer function relating gas mileage to attribute scale values in DEFINE.

provided. A transfer function relating attribute values (dollars, MPG) to a 0-100 scale is then displayed to the user, and if the user indicates that the function accurately reflects his or her attitudes, then the next dimension is scaled. An example transfer function for a hypothetical user's scaling of gas mileage is shown in Figure 1.

Object- and phrase-anchored scales. Dimensions that cannot easily be described quantitatively, such as car size, comfort, color, safety, maintenance, and make, can be scaled with reference to specific phrases or objects. For example, size might be described as subcompact, compact, intermediate, or full-size; color might be scaled with respect to white, black, red, yellow, blue, and green; safety and maintenance scales might use consumer ratings as reference scale points; and make of the automobile might be a personalized scale of manufacturers. DEFINE allows up to 10 levels (objects or phrases) per scale, and the user is asked to input the phrases or objects in an ascending order, from worst to best. The first level is assigned a scale value of 0 and the last level is assigned a value of 100 . The user is prompted to provide scale values between 0 and 100 for intermediate attribute levels. The hypothetical user evaluating cars might scale the color dimension in the following manner (scale values are given in parentheses after the color identifier): white $(0)$, black $(10)$, red (40), yellow (45), blue (80), and green (100).

\section{Response Elicitation}

After the problem set space has been defined, a series of exemplars, composed of randomly selected values, phrases, or objects from each attribute dimension, are presented to the user for evaluation. Users may either rate the exemplar on a $0-100$ scale, or judge that a particular example could not actually occur and therefore cannot be rated (e.g., a subcompact car for $\$ 15,000$ that is uncomfortable and gets 15 MPG). Ratings of exemplars are indicated by moving an arrow across a rating scale and pressing ENTER when the arrow is at the desired position. Exemplar ratings and their dimensional scale values are then stored in a file, and the exemplar number and its rating are displayed at the left of the screen. While rating subsequent exemplars, previously rated exemplars may be brought back to the screen for comparison purposes, which affords the user an opportunity to contemplate subtle trade-offs between different attributes as well as increases the reliability of the user's ratings. Figure 2 shows a typical display in which Exemplar 8 is being rated (41.80). Exemplar 2 is not represented in the "OLD PROFILES" window because it was judged to be impossible, but it may still be recalled for comparison purposes.

The sequence of rating exemplars interspersed with reviewing previously rated exemplars continues until the user terminates the program. The user is then asked if the definition of problem domain should be saved for future use, and if so, to provide a definition name.

\section{PROGRAM SOLVE}

SOLVE is essentially a regression program that constructs a multiplicative weighting function that minimizes the sum of squared errors between ratings provided by the user and model predictions. Starting with an arbitrary initial model, the program applies a reiterative method of gradient descent through the multidimensional weighting space to produce a model of minimal error (sum of squared differences between model predictions and elicited ratings). Before describing the program further, a brief discussion of the role of linear models of human judgment is in order.

Linear models have been used to aid, represent, and even replace decision-makers in a variety of situations. The models have performed well where it has been possible to compare human responses to linear models representing these responses on the basis of predictiveness to some objective criterion. Indeed, the strength of linear models creates a situation in which sizeable changes in attribute weights have only a limited impact on model validity (Dawes \& Corrigan, 1974).

In the present situation, however, there is no external criterion for validating the derived model; the model is valid only to the degree that its application imitates the judgmental policy inherent in the personal preferences expressed by the user. Three forms of multiattribute utility models-additive, multiplicative (quasi-additive), and multilinear-have received the most attention for representing human preferences (Fischer, 1979).

Additive utility models assume that the overall utility change in an alternative due to changing a single attribute from its least to its most desired level remains constant, regardless of the status of other attributes. Since only $n$ constants (attribute weights) need to be derived for an additive model of an $n$-dimensional problem, model 




Figure 2. Display screen presented by DEFINE during response elicitation.

construction is simplified, but possible interactions between dimensions are completely ignored. Multiplicative models are a special case of the general multilinear model, to be described below, but require $n+1$ scaling constants to be derived in order to model an $n$-dimensional problem. The additional constant is a global index of the substitutability among the attributes, and operates in the model equation to approximate interaction effects. Multilinear utility models are full regression equations in which each main effect and interaction term has an independent scaling constant. While providing the optimal linear fit, complete multilinear models require the derivation of $2^{n}-1$ constants for an $n$-dimensional problem.

As stated earlier, SOLVE uses a reiterative method of gradient descent to derive a multiplicative utility function from ratings provided by the user. Unfortunately, the extensive use of recursive computations requires considerable time for program execution. That is, increasing problem dimensionality increases program execution time exponentially, due to the fact that every iteration of the program differentiates each attribute scale constant with respect to each of the other dimension weights (and the global constant of substitutability) for every data point being used in the model construction. As might be expected, since the differentiation described above must be computed for each data point used in the model construction, increasing the number of exemplars and their ratings increases the time required for the program to execute in a roughly linear manner. Figure 3 illustrates the time needed for program execution for problems of different dimensionality using different numbers of data points. These simulations (computer-generated data with random perturbations) were run on a Dell System 310 computer with an 80386 microprocessor with 32-bit architecture running at $20 \mathrm{MHz}$.

SOLVE modifies the weights, or scale constants, of the problem dimensions with each iteration of the program so that predictions of the new model more closely approximate the provided ratings. Reiteration continues until a predefined iteration limit is surpassed, the model fits the data within some specified error tolerance, or modifications of the model from one iteration to the next are insignificant (i.e., changes in the fourth and fifth decimal places). Convergence to the best "fitting" model is strongly influenced by the "fit" of the initial starting point as well as by the amount of noise, or unreliability, inherent in the ratings.

\section{DISCUSSION}

Representing preference policies within a multidimensional problem domain with a quantitative model affords special opportunities for both users and systems designers. The requirements of defining and scaling relevant problem dimensions and the exercise of rating hypothetical situa-

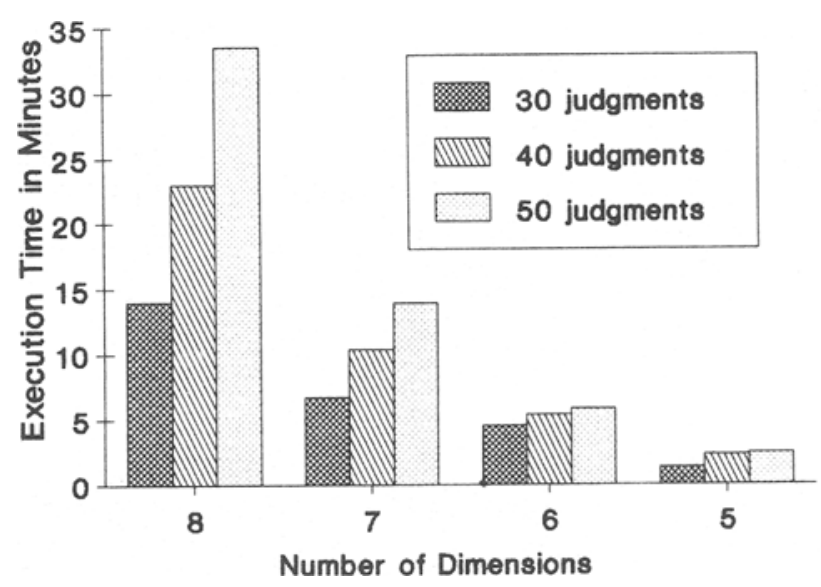

Figure 3. Execution time of SOLVE as a function of problem dimensionality and number of data points used in model derivation. 
tions can be helpful in clarifying the user's thoughts about the trade-offs involved in difficult situations. Perhaps even more importantly, once a valid model of the user's preferences has been derived, it may be used to assist the application of his or her policy in a more effective manner than might otherwise be possible. In situations in which time is limited or where the number of alternatives (or attributes per alternative) is large, the quantitative model could be used as a filter to order alternatives. This would allow the user more effectively to focus on the "better" alternatives currently available. In addition, when the user must interact with an external system (e.g., an energy or ecological system) that has been or could be quantitatively modeled, the preference model of the user can be applied not only to the current policy alternatives, but also to the future results predicted by the external model. Thus, not only can the application of a quantitative preference model assist with the selection of current choices to achieve shortterm goals, but alternatives to achieve long-term goals can be evaluated as well (Hammond, Klitz, \& Cook, 1977).

While it cannot be inferred that preference models simulate the process by which decision-makers reach their decisions, the model can be very useful in understanding how various attributes influence the decision-makers' judgments. In the present case, the multiplicative utility function derives a global constant that indexes the substitutability of the various attributes of holistic judgments. In some circumstances, depending on the dimensions of the problem, this index might be seen as an index of risk aversion, that is, the degree to which an individual is willing to let high attribute levels in one dimension compensate for low values in another.

An interesting application that may be possible with the type of modeling described here is the development of an on-line, real-time evaluator for training systems operators. If an expert, for example a flight instructor, were to provide an explicit model of his or her evaluation policy for integrating flight performance parameters such as airspeed, altitude, heading, and so forth, then it would be relatively easy to provide student pilots with continuous feedback on their overall flight performance by putting current flight data into the model and displaying the result. In addition to the potential benefits attained by the overall performance feedback, it might be possible to use the dimension weights of the model and the current attribute values to cue the student with regard to the distribution of his or her attention among the different flight performance measures to assist overall flight performance at any given point. In fact, an evaluation model similar to that described for capturing a flight instructor's evaluation policy has been constructed and has proven to be a sensitive measure of pilot performance, which offers reason for optimism that such an application might be feasible (Ross \& Mundt, 1988). The potential for using experts' models to train novice operators is currently being investigated.

Another possible application of the models derived from these programs is the measurement of attitudinal differences between different demographic groups. If differ- ent groups, such as young and old people, or experienced and inexperienced drivers, were systematically to evaluate common situations differently, then their attitudinal differences should be quantifiable with respect to the weights of the different attributes in the best fitting model. This type of attitudinal assessment has been utilized with mixed success by other investigators using different modeling forms and response elicitation techniques (Curry, 1976; Flathers, Griffin, \& Rockwell, 1982; Thurman, 1986; Yntema \& Klem, 1965).

The only real constraint on the type of models these programs derive is that each problem dimension must be utility-independent of its complement, that is, the desired direction of change in each dimension (e.g., lower price, higher gas milage, more comfort, etc.) must not depend on the value of the other attributes. While this constraint is not overly restrictive, in some situations care must be exercised in the selection of problem dimensions. It should be pointed out, however, that even in particular situations where the preferred direction of change in a given dimension changes due to special circumstances, it should be possible to construct a preference model for each class of special circumstance. Application of a production system should then make it possible to apply the appropriate model of user preference to the current circumstance (Lewis, 1985).

\section{REFERENCES}

CURRY, R. E. (1976). Worth assessments of approach to landing. Proceedings of the Annual Conference on Manual Control. (NASA Report TMX-73), 585-591. (NTIS No. N77-14745)

DAWES, R. M., \& CoRRIGAN, B. (1974). Linear models in decision making. Psychological Bulletin, 81, 95-106.

Fischer, G. W. (1979). Utility models for multiple objective decisions: Do they accurately represent human preferences? Decision Sciences, 10, $451-479$.

Flathers, G. W., Griffin, W. C., \& Rockwell, T. H. (1982). A study of decision-making behavior of aircraft pilots deviating from a planned flight. Aviation, Space \& Environmental Medicine, 53, 958-963.

Hammond, K. R., KLITz, J. K., \& COOK, R. L. (1977). How systems analysts can provide more effective assistance to the policy maker (Report No. RM-77-50). Laxenburg, Austria: International Institute for Applied Systems Analysis

KeEney, R. L., \& RAFFi, H. (1976). Decisions with multiple objectives: Preferences and value tradeoffs. New York: Wiley.

LewIS, C. M. (1985). Rule-based analysis of pilot decisions. Proceedings of the 29th Annual Meeting of the Human Factors Society, 2, 907-911

Ross, L. E., \& MUNDT, J. C. (1988). Multiattribute modeling analysis of the effects of low blood alcohol level on pilot performance. $\mathrm{Hu}$ man Factors, 30, 293-304

Thurman, Q. C. (1986). Estimating social-psychological effects in decisions to drink and drive: A factorial survey approach. Journal of the Studies of Alcoholism, 47, 447-454.

VON WINTERFELDT, D., \& EDWARDS, W. (1986). Decision analysis and behavral research. Cambridge, England: Cambridge University Press.

YNTEMA, D. B., \& KLEM, L. (1965). Telling a computer how to evaluate multidimensional situations. IEEE Transactions on Human Factors in Electronics. HFE-6, 3-13.

YNTEMA, D. B., \& TORGERSON, W. S. (1961). Man-computer cooperation in decisions requiring common sense. IRE Transactions on $\mathrm{Hu}$ man Factors in Electronics. HFE-2, 20-26. 